\title{
Baade-Wesselink Analysis of Magellanic Cloud Cepheids
}

\author{
Jesper Storm ${ }^{1,2,4}$, Bruce W. Carney ${ }^{1,2,5}$, Wolfgang P. Gieren ${ }^{1,2,3,6}$, \\ Pascal Fouqué ${ }^{7}$, Anne M. Fry ${ }^{8}$
}

\begin{abstract}
We apply the Barnes-Evans variant of the Baade-Wesselink method to Cepheids in the LMC and SMC in an attempt to determine the distance directly to individual stars in these galaxies and to determine the metallicity effect on the Cepheid period-luminosity relation. We now have $K$-band light curves for a sample of SMC stars as well as for many Cepheids in young clusters in the LMC. Using the $F_{V},(V-K)$ calibration of Fouqué \& Gieren (1997) we find preliminary evidence for a metallicity effect which makes metal poor Cepheids brighter. This is at odds with earlier results based on optical photometry and the reason is not entirely understood yet.
\end{abstract}

\section{Introduction}

Cepheids are radially pulsating variable stars and due to the simple nature of their oscillations they have become one of the most important primary distance indicators. In particular, the Hubble Space Telescope Key Project on the extragalactic distance scale has observed Cepheids in numerous distant galaxies to determine their distance through the Cepheid period-luminosity $(P-L)$ relation. These distances are in turn used to calibrate secondary distance indicators which can reach out to distances where the pure Hubble flow dominates and where the Hubble constant can be determined (e.g. Ferrarese et al. 1999).

Their goal has been to determine the Hubble constant to better than $10 \%$. However, the calibration of the $P-L$ relation depends critically on the assumed distance to the Large Magellanic Cloud. This distance is still heavily disputed and current estimates range between 18.2 and 18.7 mag. At one extreme of this range we find the value of $18.70 \pm 0.10$ mag by Feast \& Catchpole (1997) who calibrated the Cepheid $P-L$ relation using Hipparcos parallaxes to galactic Cepheids. At the other extreme we find the value of $18.22 \pm 0.13 \mathrm{mag}$ by Udalski et al. (1998) who argue for an additional reddening correction to the original

\footnotetext{
${ }^{1}$ Visiting Astronomer, Cerro Tololo Inter-American Observatory. CTIO is operated by AURA, Inc. under cooperative agreement with the National Science Foundation

${ }^{2}$ Visiting Astronomer, Las Campanas Observatory

${ }^{3}$ Visiting Astronomer, European Southern Observatory

${ }^{4}$ Astrophysikalisches Institut Potsdam, An der Sternwarte 16, D-14482 Potsdam, Germany

${ }^{5}$ University of North Carolina at Chapel Hill, North Carolina, USA

${ }^{6}$ Universidad de Concépcion, Concépcion, Chile

${ }^{7}$ European Southern Observatory, Santiago, Chile

${ }^{8}$ Case Western Reserve University, Ohio, USA
} 
result by Guinan et al. (1998) who found $18.30 \pm 0.07 \mathrm{mag}$ using an eclipsing binary in the LMC. Many more results falls in between, but no single result appears decisively superior than the rest for the moment.

An additional assumption is that the $P-L$ relation is independent of the metallicity of the Cepheids in question. This assumption also remains a matter of dispute from the empirical point of view where Kennicutt et al. (1998) finds a small effect and Sasselov et al. (1998) find a large effect, both in the sense that metal poor Cepheids are fainter than metal rich ones. On the theoretical side a similar controversy exists which was discussed at this meeting by Marconi, Caputo, \& Ripepi (2000), who found an effect in the opposite sense, and by Alibert \& Baraffe (2000).

\section{The Barnes-Evans Method}

The Barnes-Evans surface brightness variant of the Baade-Wesselink method is a geometric method for determining the distance to a radially pulsating star. It relates geometrically the angular diameter, $\theta$, of the star at a given phase, $\phi$, the radius, $R(\phi)$, of the star and the distance, $d$, through the relation:

$$
d \times \theta(\phi)=2 \times R(\phi)=2 \times\left(R_{0}+\Delta R(\phi)\right) .
$$

The angular diameter is related to the visual surface brightness through the relation $F_{V}=4.2207-0.1 V-0.5 \log \theta . F_{V}$ has recently been calibrated by several workers. We adopt here the calibration by Fouqué \& Gieren (1997) which is based on the recent accurate interferometric measurements of radii of giants and supergiants. They found $F_{V}=3.947-0.380\left(V-R_{J}\right)$ and $F_{V}=$ $3.947-0.131(V-K)$ respectively. $\Delta R(\phi)$ can be determined by integrating the radial velocity curve and with an observed color curve in $V-R_{J}$ or $V-K$ we can build up a dataset of $(\theta(\phi), \Delta R(\phi))$ pairs and by linear regression or some other procedure we can fit these data and solve Eq. 1 for the distance $d$ and the mean radius $R_{0}$.

It should be stressed that this calibration is entirely independent of the other classical calibrations of the zero-point of the Cepheid $P-L$ relation. In particular it is independent of the distance estimates to galactic open clusters and of the distance to the LMC.

\section{The Samples}

To address the issue of the distance to the LMC we are attempting to apply the Barnes-Evans method to a number of LMC cluster Cepheids. The LMC contains a number of populous blue clusters which contain numerous Cepheids (Storm et al. 1988; Mateo 1992). Data are already available for six Cepheids in NGC 1866 and more is on the way for NGC 2031 and NGC 2136. In this way we will get a direct measure of the distance to the LMC as well as a direct measure of the internal accuracy of the method. These Cepheids are all of short period, $3-5 \mathrm{~d}$, and are thus reasonably easy get good phase coverage for. However, they are quite faint in the near-IR, $K \approx 14.5 \mathrm{mag}$ and a significant fraction of Cepheids in this period range can be expected to be overtone pulsators (see 
e.g. Alcock et al. 1999). Gieren, Fouqué, \& Storm (1999) have presented a first analysis of the star HV12198 in NGC 1866 and in the following we will concentrate on the SMC efforts.

To address the question of the metallicity dependence of the $P-L$ relation the SMC Cepheids provides a good baseline as they are fairly metal poor compared to the galactic Cepheids. Luck et al. (1998) find a metallicity difference of $\Delta[\mathrm{Fe} / \mathrm{H}]=-0.7$.

We are studying a sample of five Cepheids with periods of about $15 \mathrm{~d}$. They are reasonably bright and thus easy to observe photometrically with 1-m class telescopes. The long periods avoid the potential problem of overtone pulsators, but at the expense of making it harder to obtain good phase coverage.

For all these stars we have radial velocity measurements and infrared light curves from Las Campanas, Cerro Tololo and La Silla observatories as well as optical light curves from Las Campanas and Cerro Tololo.

The sampling is currently very good for the SMC stars while it is still a bit sparse for most of the LMC stars.

\section{Applying the Barnes-Evans Method to the SMC Stars}

\subsection{The Effect of Metallicity on the Calibration Itself}

The empirical calibration of the Barnes-Evans method by Fouqué \& Gieren was carried out using galactic Cepheids with approximately solar metallicity. As the SMC Cepheids have a significantly lower metallicity we cannot apply the $F_{V}$ calibration directly but will first have to evaluate the metallicity effect on the calibration. We expect that there can be a significant effect as the calibration is largely a temperature calibration based on the color index.

Using Kurucz model atmospheres at fixed gravity we have differentially modified the calibration of Fouqué \& Gieren (1997) to correspond to a range of metallicity values. For the $F_{V},\left(V-R_{J}\right)$ calibration, the effect is of the order $\Delta M_{V}=-0.2 \mathrm{mag} / \mathrm{dex}$ in the sense that the distance to a metal poor Cepheid will be overestimated if the uncorrected calibration is applied. For the $F_{V^{r}},(V-K)$ relation, the metallicity effect is negligible which is an additional bonus for this version of the Barnes-Evans method.

\subsection{The $\left(V-R_{J}\right)$ Calibration}

In addition to the metallicity effect, the $F_{V},\left(V-R_{J}\right)$ calibration suffers from several other problems. Gieren, Fouqué, \& Gómez (1998) find in particular that the distances based on this calibration to a sample of open clusters Cepheids are systematically longer than the $F_{V},(V-K)$ based results by $16 \%$, which leads to distances which are too long by $0.32 \mathrm{mag}$. They prefer the $(V-K)$ based calibration as it shows significantly less scatter and the fits to Eq. 1 were much better defined, thus much less dependent on the actual fitting procedure.

For all these reasons we have decided to complement the optical light curves with $K$ band light curves to take full advantage of the method. 

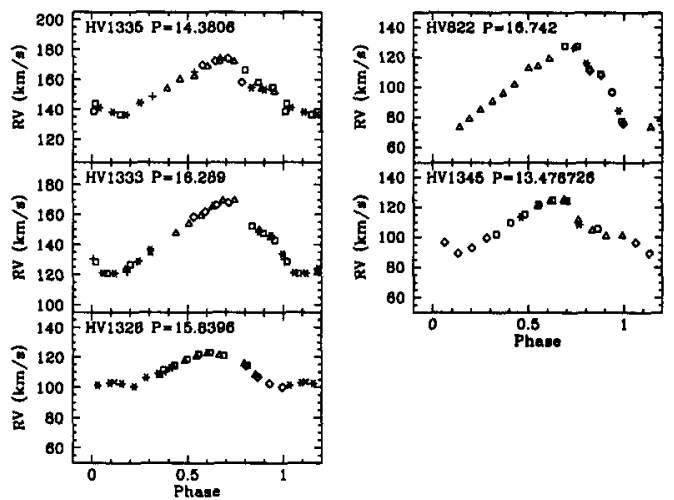

Figure 1. Radial velocity curves for the SMC Cepheids in this study. The different symbols correspond to different observing runs. Note that the velocity scale is the same for all the plots, only the zero point is different.

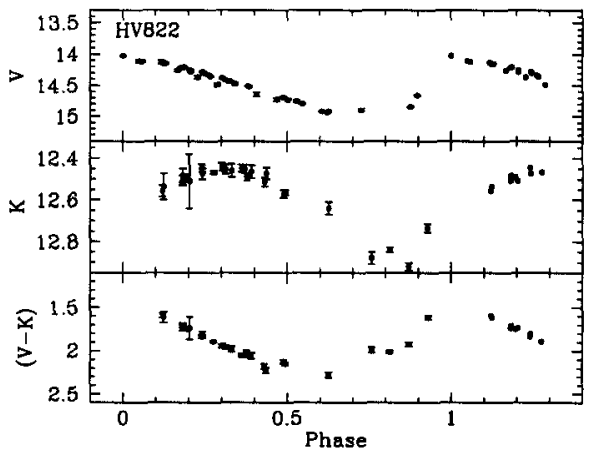

Figure 2. The $V$ and $K$-band light curves and the $V-K$ color curve for $\mathrm{HV} 822$.

\section{HV822 as an Example}

To allow a direct comparison with the galactic Cepheids studied by Fouqué \& Gieren (1997) we adopt the same fitting procedure as they have used, i.e. the so called "inverse fit" where the angular diameter is the dependent variable in the linear regression.

We have adopted the reddenings determined by Laney \& Stobie (1994) except for HV822 which is not in their list. We have adopted a value of $E(B-V)=0.06$ as representative for this location in the SMC which is the same value as adopted for the other Cepheids.

In Fig. 1 we have plotted the radial velocity curves for the SMC Cepheids which we have studied. It can be seen that the phase coverage is quite good and the curves are very well defined. Note also the very different shapes of the 
radial velocity curves in spite of the fact that the periods are almost the same for these stars.

In Fig. 2 we have plotted the $V$ and $K$-band light-curves as well as the $V-K$ color curve for HV822. The $V$-band coverage is reasonable but the $V-K$ coverage in the region 0.45 to 1.0 is not quite good enough.

When we perform the Baade-Wesselink analysis for the $\left(V-R_{J}\right)$ version of the method we find a distance modulus of $18.90 \pm 0.07$. We have not corrected the value for the systematic offset found by Gieren et al. (1998). The fit based on the $V-K$ data is shown in Fig. 3 and the result is $(m-M)_{0}=19.28 \pm 0.14$. If, however, we exclude the region with poor coverage we get $(m-M)_{0}=$ $19.19 \pm 0.12$.
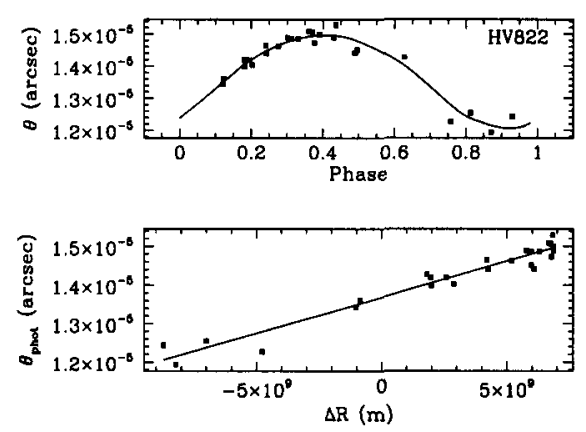

Figure 3. The upper panel shows the angular diameter as a function of phase. The points represents the photometrically determined angular diameter and the solid curve represents the integrated radial velocity curve at the best fitting distance. The lower panel shows the fit of angular diameter versus radius change (Eq. 1). This is the fit using the $V-K$ data.

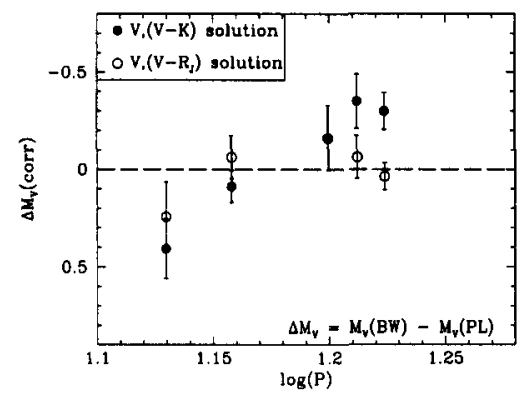

Figure 4. The difference in absolute magnitude between the BaadeWesselink (BW) and period-luminosity based estimates. 


\section{The Metallicity Effect on the $P-L$ Relation}

To quantify the metallicity effect on the $P-L$ relation we will need to determine the difference in absolute magnitude between the Baade-Wesselink results for the SMC stars and the predicted value from the $P-L$ relation, or $\Delta M_{V}=$ $M_{V}(\mathrm{BW})-M_{V}(P-L)$.

As the $P-L$ relation is a statistical relation we cannot expect the Cepheids to lie exactly on the $P-L$ relation, and as we only have a few stars in the SMC we will have to evaluate the systematic offset from the $P-L$ ridge line. We do that by comparing the observed visual magnitude with the Laney \& Stobie (1994) ridge line after we have corrected for depth effects following their method. The offset for each of the stars is then applied to the Baade-Wesselink absolute magnitudes to bring them on the ridge line as well.

We compute $\Delta M_{V}$ for all the stars for both the $V-R_{J}$ and $V-K$ based calibrations and plot the results in Fig. 4. Notice that we have not applied any correction to bring the $V-R_{J}$ results into agreement with the $V-K$ results. Still, we do not see a systematic difference between the two calibrations, so here we are at odds with Gieren et al. (1998). The reason for this disagreement is not yet clear, but we can hardly justify applying such a correction. Consequently, these new results also disagree with the preliminary results from Storm et al. (1999) based only on the $V-R_{J}$ calibration and including the said correction.

From Fig. 4 we note that there is still significant scatter between the results from the different calibrations and for the different stars. We are still working on completing the analysis of the $K$-band calibration data as well as on improving the statistical treatment of the data entering the fit, and this might improve on the situation. The overall metallicity effect seems to be small and the sign might even be opposite of what was previously suggested, i.e. the metallicity effect could increase the inferred distance to the Magellanic Clouds. This would be counter to our expectations as this would increase the disagreement with several non-Cepheid based distance determinations.

\section{Conclusions}

The systenatic offset between the $V-R_{J}$ and $V-K$ based Barnes-Evans results as found by Gieren et al. (1998) cannot be reproduced here.

We now find an indication of a possibly small correction to the $P-L$ relation in the opposite sense as previously found, i.e. that metal poor stars will be brighter than metal rich stars. This result is still preliminary pending a proper statistical analysis of the individual fits, but it is in qualitative agreement with the theoretical computations by Marconi et al. (2000).

\section{References}

Alcock, C., Allsman, R. A., Alves, D., et al. 1999, ApJ, 511, 185

Alibert, Y. \& Baraffe, I. 2000, in these proceedings, p. 250

Feast, M. W. \& Catchpole, R. M. 1997, MNRAS, 286, L1

Ferrarese, L., Gibson, B. K., Kelson, D. D., et al. 1999, astro-ph/9909134 
Fouqué, P. \& Gieren, W. P. 1997, A\&A, 320, 799

Gieren, W. P., Fouqué, P., \& Gómez, M. 1998, ApJ, 488, 74

Gieren, W. P., Fouqué, P., \& Storm, J. 1999, poster presented at IAU Coll. 176, not appearing in these proceedings

Guinan, E. F., Fitzpatrick, E. L., DeWarf, L. E., et al. 1998, ApJ, 509, L21

Kennicutt, R. C, Stetson, P. B., Saha, A., et al. 1998, ApJ, 498, 181

Laney, C. D. \& Stobie, R. S. 1994, MNRAS, 266, 441

Luck, R. E., Moffett, T. J., Barnes, T. G., \& Gieren, W. P. 1998, AJ, 115, 605

Marconi, M., Caputo, F., \& Ripepi, V. 2000, in these proceedings, p. 338

Mateo, M. 1992, PASP, 104, 824

Sasselov, D. D., Beaulieu, J.P., Renault, C., et al. 1998, A\&A, 324, 471

Udalski, A., Pietrzyński, G., Woźniak, P., et al. 1998, ApJ, 509, L25

Storm, J., Andersen, J., Blecha, A., \& Walker, M. F. 1988, A\&A, 190, L18

Storm, J., Carney, B. W., \& Fry, A. 1999, in ASP Conf. Ser. Vol. 167, Harmonizing Cosmic Distance Scales in a Post-Hipparcos Era, ed. D. Egret \& A. Heck (San Francisco: ASP), 320

\section{Discussion}

Pawel Moskalik: What is the accuracy of your velocity measurements?

Jesper Storm: About 1-2 $\mathrm{km} \mathrm{s}^{-1}$.

Dave Laney: What is the systematic difference between the radii of galactic and SMC Cepheids? This is a simpler comparison than dealing with absolute magnitudes and less dependent on theory.

Jesper Storm: We have not yet made this comparison. 